\title{
Effect of Coronary Artery Bypass Grafting on the Plasma Levels of VEGF, bFGF, Copper and NO as Angiogenic Biomarkers in Chronic Ischemic Heart Disease Patients
}

\author{
Ahmed Farouk ${ }^{1}$ and Mohammed H Hassan ${ }^{2}$ \\ ${ }^{1}$ Department of Cardiothoracic Surgery, Assiut University Hospitals, Assiut, Egypt \\ ${ }^{2}$ Department of Medical Biochemistry and Molecular Biology, Qena Faculty of Medicine, South Valley University, Qena, Egypt
}

Corresponding author: Dr. Mohammed H Hassan, Lecturer of Medical Biochemistry and Molecular Biology, Qena Faculty of Medicine, South Valley University, Qena, Egypt, Tel: 201098473605; E-mail: mohammedhosnyhassaan@med.svu.edu.eg

Received: 16 May 2016; Accepted: 26 July 2016; Published: 30 July 2016

Citation: Farouk A, Hassan MH. Effect of Coronary Artery Bypass Grafting on the Plasma Levels of VEGF, bFGF, Copper and NO as Angiogenic Biomarkers in Chronic Ischemic Heart Disease Patients. Ann Clin Lab Res. 2016, 4: 3.

\section{Abstract}

Objectives: Myocardial ischemia offers paradoxical protection from ischemic necrosis by the development of coronary collateral circulation through the process of angiogenesis. The current study aimed to assess the plasma levels of vascular endothelial growth factor "VEGF", basic fibroblast growth factor "bFGF", nitric oxide "NO" and copper as angiogenic biomarkers to evaluate their role in the development of coronary collateral circulation in patients with chronic ischemic heart disease. Also, to evaluate the angiogenic modulator effects of coronary artery bypass grafting (CABG) via repeated measurements of such biomarkers 3 months postoperative.

Methods: This case control and observational study involved 25 ischemic heart disease male patients candidate for elective coronary artery bypass grafting who admitted at the department of cardiothoracic surgery, faculty of medicine, Assiut University and 25 healthy age matched males as control group. Spectrophotometric assay of NO and copper and ELISA assay of VEGF and bFGF plasma levels were done.

Results: Significant higher plasma VEGF, bFGF, copper and NO levels preoperatively when compared with the control group ( $<<0.01$ for all) with significant lowering of their plasma levels when repeatedly measured three months following the CABG ( $p<0.01$ for all).

Conclusions: Our findings revealed that plasma levels of VEGF, bFGF, copper and NO have been significantly decreased in patients with chronic myocardial ischemia following $C A B G$, thus, proving the proper coronary reperfusion effect of CABG with decrease the demand for the angiogenesis in the ischemic myocardium. There was a positive correlation between the plasma levels of VEGF and NO.
Keywords: Angiogenesis; VEGF; bFGF; copper; NO; CABG

\section{Introduction}

Coronary artery bypass grafting (CABG) is characterized as "open-heart surgery" in which a part of a blood vessel is grafted from the aorta to the coronary artery to bypass the occluded part of the coronary artery and enhance the blood supply to the heart, so the basic premise of CABG is to restore perfusion to the myocardium [1].

Angiogenesis, also called neovascularization, happens in the healthy body for mending wounds and for restoring blood supply to the damaged tissue. Angiogenesis assumes a part in the reaction to ischemia by the heart muscle. Some diseases are augmented during pathologic circumstances by increased vascular growth (e.g. tumors), whereas in others, insufficient vascular growth adds to morbidity and mortality (e.g. ischemic heart disease) [2]. Myocardial ischemia provides paradoxical protection from ischemic necrosis by formation of coronary collateral circulation [3], which helps the newly formed vessels to supply the ischemic tissue [2]. A biochemical effect produced by the ischemic myocardium has been found to initiate the events leading to DNA formation and to mitosis in the collateral vessels [4]. The best known growth factors with demonstrated angiogenic power are vascular endothelial growth factor (VEGF) and basic fibroblast growth factor (bFGF) [5].

The VEGF family formed of five secreted dimeric glycoprotein growth factors in mammals, VEGF (or VEGF-A), (VEGF-B), (VEGF-C), (VEGF-D) and PIGF (placental growth factor). VEGFs related to the platelet derived growth factor PDGF/VEGF superfamily of growth factors, all having VEGF/ PDGF homology domain with eight conserved cysteine residue included in inter- and intra-chain disulfide linkage formation. The VEGF ligands are significant controllers of blood and lymphatic vessel formation and growth [6]. VEGF, the original angiogenic growth factor, was initially recognized as a permeability-inducing factor discharged by tumor cells [7], and later as a growth factor for vascular endothelial cells [8]. 
Notwithstanding working as a mitogen for endothelial cells "ECs", VEGF also control the EC survival [9], intervened through the PI3K-Akt pathway and by enhancing the expression of anti-apoptotic proteins [10]. VEGF is also a strong enhancer of vascular permeability and inflammation [11]. Akt additionally phosphorylates and activates eNOS, empowering thusly vasodilatation, permeability and angiogenic processes [12].

Fibroblast growth factor-2, also known as basic fibroblast growth factor, is 18 Kilo Dalton "KD", single chain peptide with broad mitogenic capacity. It is formed by, and can act directly upon, vascular endothelial and smooth muscle cells, and functions as angiogenic factor [13].

Copper "Cu" is an essential trace metal that can be presented as oxidized $\mathrm{Cu}$ (II) or reduced $\mathrm{Cu}$ (I) states, allowing the metal to perform a vital role in cell physiology as a catalytic cofactor [14]. The effect of copper in angiogenesis was initially noted in 1980 when, while attempting to segregate a peptide "endothelial stimulating growth factor", Mc Auslan and Reilly found that copper salts were the least complex angiogenic parts of tumor extracts [15], stimulating the migration of endothelial cells in vitro. Copper was found to activate the multiplication and migration of endothelial cells in vitro [16].

Nitric oxide "NO" was found to be a strong vasodilator in 1979 [17], and later known as an endothelium relaxing factor [18]. This simple molecule is a short-lived free radical [19]. Some activities of nitric oxide are linked to its intracellular second messenger nature, and other effects result from its paracrine actions, occurred through activation of the guanylate cyclase/3', 5' cyclic-guanosine monophosphate (GC/cGMP) pathway [20]. NO is formed through conversion of L-arginine and molecular oxygen to L-citrulline via the action of nitric oxide synthases (NOS) [21]. Three isoforms of NOS has been known with the different sites and regulation. They involve neuronal NOS (nNOS; also known as NOS-1), inducible NOS (iNOS; also known as NOS-2) and endothelial NOS (eNOS; also known as NOS-3). The eNOS is the membrane bound isoform first seen in the vascular endothelial cells (VEC), while the nNOS is the isoform present in the cytosol and first seen in the neuronal tissues. The iNOS can be inducible in various cells including vascular cells, tumor cells and macrophages [21]. The changes of the systemic levels of VEGF, bFGF, copper and NO as angiogenic biomarkers in chronic ischemic heart disease patients especially after revascularization intervention are largely unknown. Published data is scarce and only describes short-term changes of some of these angiogenic biomarkers circulating levels after percutaneous coronary intervention $(\mathrm{PCl})$ or bypass surgery, hence the present study was done to evaluate the effect of CABG on the systemic levels of such angiogenic biomarkers in patients with chronic myocardial infarction.

\section{Methods}

\section{Study group}

This study was a prospective case control and clinical observational study and it was carried out on 25 ischemic heart disease male patients with multiple partial or complete vessel occlusions with failure of medical therapy and failure of cardiac catheterization and stent selected as candidate for elective coronary artery bypass grafting who admitted at the department of Cardiothoracic surgery, faculty of medicine, Assiut University, after obtaining approval of university hospital ethics committee and informed consent from the included patients. Patients with previous cardiac surgery, ejection fraction less than $40 \%$, chronic renal insufficiency (serum creatinine $>1.7 \mathrm{mg} / \mathrm{dl}$ ) or renal failure on dialysis or use of steroids were excluded from the study. This is in addition to 25 apparently healthy age matched males selected as control group. The study was carried out during the period from July 2014 to July 2015. No deaths in the studied patients and all the patients involved completed the study.

\section{Data collections}

For each included patient, complete history taking, general and systemic examination, E.C.G, chest X-ray and echocardiography were done; hemodynamic data were recorded as regard heart rate, arterial blood pressure and central venous pressure preoperative, one hour and six hours postoperative.

\section{Laboratory methods}

In addition to routine blood analysis preoperatively in the form of complete blood count, liver and kidney function tests, coagulation profile, fasting blood sugar, serum electrolytes in the form of sodium, potassium, calcium and magnesium, three $\mathrm{mls}$ venous blood was drawn from all patients (preoperative and three months postoperative) and control group, on EDTA tubes, for assay VEGF, bFGF, copper and nitric oxide, they were then centrifuged at $3500 \mathrm{rpm}$ for $15 \mathrm{~min}$ at $4^{\circ} \mathrm{C}$ and the plasma were transferred into $1 \mathrm{ml}$ cryotubes, and stored at $-80^{\circ} \mathrm{C}$ for later analyses. The laboratory measurements were done at the Metabolic and Genetic Disorders Unit, faculty of medicine, Assiut University. Using commercially available assay kit according to manufacturer protocol for measurements of:

Plasma human VEGF (supplied by Elabscience Biotechnology Co., Ltd, Catalog No: E-EL-H2164). It is reported that the studies done using serum assays of VEGF should be interpreted with some caution, as serum levels were expressed as mean values \pm standard deviation, but VEGF is not a normally distributed marker, also measurement of VEGF in serum can produce erroneous levels of VEGF and it has been recommended that plasma samples should be used [22]. In fact, estimation of serum VEGF would significantly overestimate the actual levels of free VEGF as some VEGF is discharged from platelets when blood clumps, and so, serum VEGF levels may (artefactually) rise significantly overtime after 
clotting occurs [23]. Plasma human bFGF (supplied by Elabscience Biotechnology Co., Ltd, Catalog No: E-EL-H0483). Both VEGF and bFGF were measured using an enzyme-linked immune-sorbent (ELISA) assay kits based on sandwich-ELISA principle by enzyme-linked immune-sorbent assay (ELISA) multiskan EX microplate photomter, thermo scientific, STAT FAX-2100, USA.

Plasma copper (supplied by Spectrum Diagnostics, Catalog No: 232 001). The assay based on that copper forms with 4(3,5-dibromo-2-pyridilazo)- $\mathrm{N}$-ethyl-sulfopropylaniline a chelate complex. The increase of absorbance of this complex can be measured and is proportional to the concentration of total copper in the sample. Plasma nitric oxide (supplied by Biodiagnostics, Cairo, Egypt). The assay based on that in acid medium and in the presence of nitrite (endogenous nitrite concentration as indicator of nitric oxide production) the formed nitrous acid diazotise sulphanilamide and the product is coupled with $\mathrm{N}$-(1-naphthyl) ethylene diamine. The resulting azo dye has a bright reddish-purple color which can be measured. Both copper and NO were measured using T60 UV visible spectrophotometer. PG INSTRUMENTS LIMITED, alma park wibtoft, Leicester shreshire, England. LE17SBE. Serial No. 20-1650-01-0010.

\section{Operative procedure}

All patients were approached via a median sternotomy approach. Standard aortic and biacaval cannulation was done. An initial dose of $400 \mu \mathrm{g} / \mathrm{kg}$ heparin was used to obtain an activated clotting time. Range blood pressure of $50-70 \mathrm{mmHg}$ and blood flow 2-2.4 L/min. Priming solution contains mannitol and heparin. Hypothermia for 25-30 minutes. Cold $\left(40^{\circ} \mathrm{C}\right)$ crystalloid cardioplegic arrest was used after aortic cross clamping. Coronary bypass grafting was done using venous and arterial conduits. Flow up in the I.C.U for the hemodynamic changes in the heart rate, arterial blood pressure and central venous pressure. Antipyretics and potent antibiotic therapy were given. Before discharge the patient must meet the followings: aware, impulsive breathing, detached endotracheal tube.

\section{Statistical analysis}

Statistical analysis was performed using statistical package for the social sciences (SPSS-version 17) software. The results were expressed as mean \pm standard deviation. One way ANOVA (analysis of variance) test was used to compare more than two groups as regard quantitative variable (LSD=least significant difference). Pearson correlation analysis was used to evaluate the correlations between different parameters. $P$ values of less than 0.05 were considered significant.

\section{Results}

Regarding the baseline characteristics of the included patients and duration of tracheal intubation, stay at ICU and ward, all patients were males with their mean age were 61.36 \pm 8.86 years and their mean body mass index "BMI" was 23.84 \pm 2.57 . The mean surgery time was $386.21 \pm 9.22$ minutes and the mean duration of tracheal intubation was $9.06 \pm 0.97$ hours, whereas the mean intensive care unit "I.C.U" stay and ward stay were $2.57 \pm 0.51$ and $13.14 \pm 1.51$ days respectively.

Table 1 revealed significant hemodynamic changes regarding the mean \pm SD of the heart rate, arterial blood pressure and central venous pressure, preoperative versus postoperative recordings (one hour and six hours postoperative). The mean heart rate was significantly higher one hour postoperative when compared with the heart rate preoperative and six hours postoperative $(p<0.01)$. The mean arterial blood pressure was significantly lower Six hours postoperative when compared with the preoperative and one hour postoperative $(p<0.01)$. The mean central venous pressure was significantly lower one hour postoperative versus the preoperative and six hours postoperative $(p<0.01)$.

Table 1 Mean \pm SD of some hemodynamic parameters of the patients preoperative, one hour and six hours postoperative, using one way analysis of variance "ANOVA" followed by LSD multiple comparisons

\begin{tabular}{|c|c|c|c|c|c|c|}
\hline Hemodynamic Parameters & Before & One Hour & Six Hours & P1 & P2 & P3 \\
\hline Heart Rate "Beat/min" & $89.07 \pm 3.99$ & $98.5 \pm 3.63$ & $88.43 \pm 4.57$ & $0.000^{\star *}$ & 0.598 & $0.000^{* *}$ \\
\hline Arterial Blood Pressure "mmHg" & $82.86 \pm 3.32$ & $75.07 \pm 4.55$ & $65.36 \pm 3.99$ & $0.000^{\star *}$ & $0.000^{* *}$ & $0.000^{* *}$ \\
\hline Central Venous Pressure "mmHg" & $9.62 \pm 0.45$ & $8.52 \pm 0.44$ & $8.86 \pm 0.42$ & $0.000^{* *}$ & $0.000^{* *}$ & $0.003^{* *}$ \\
\hline
\end{tabular}

$\mathrm{P} 1=$ before versus one hour; $\mathrm{P} 2=$ before versus six hours; $\mathrm{P} 3=$ one hour versus six hours. * indicate significant change at $\mathrm{P}<0.05$; ${ }^{*}$ indicate significant change at $p<0.01 ;{ }^{* * *}$ indicate significant change at $p<0.001$. NS means non-significant $(p>0.05)$.

Comparison of the mean \pm SD of the plasma levels of Comparison of the mean \pm SD of the plasma levels of VEGF, BFGF, NO and copper preoperative and postoperative versus the control group were presented in Table 2, which revealed significant higher plasma VEGF, bFGF, NO and copper levels preoperatively when compared with the control group with significant lowering of their plasma levels following the coronary artery bypass grafting but not reach to the control levels. 
Positive correlation between the plasma levels of nitric oxide and VEGF was present as shown in Figure 1.

Table 2 Comparison of the mean \pm SD of the plasma levels of VEGF, BFGF, NO and copper preoperative and postoperative versus the control group, using one way analysis of variance "ANOVA" followed by LSD multiple comparisons

\begin{tabular}{|c|c|c|c|c|c|c|}
\hline \multicolumn{7}{|c|}{ Patients $(n=25)$} \\
\hline $\begin{array}{l}\text { Biochemical } \\
\text { parameters }\end{array}$ & $\begin{array}{l}\text { Control group } \\
(n=25)\end{array}$ & Preoperative & Post-operative & P1 & P2 & P3 \\
\hline VEGF, "pg/ml" & $96.45 \pm 8.29$ & $583.11 \pm 65.54$ & $146.16 \pm 19.95$ & $0.000^{* *}$ & $0.000^{* *}$ & $0.000^{* *}$ \\
\hline bFGF, "pg/ml" & $112.29 \pm 6.26$ & $563.48 \pm 63.46$ & $155.55 \pm 13.04$ & $0.000^{* *}$ & $0.000^{* *}$ & $0.000^{* *}$ \\
\hline NO, "ng/ml" & $96.4 \pm 16.73$ & $288.57 \pm 84.19$ & $215.86 \pm 60.46$ & $0.000^{\star *}$ & $0.000^{* *}$ & $0.000^{* *}$ \\
\hline 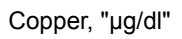 & $184.2 \pm 16.97$ & $660.71 \pm 127.13$ & $233.71 \pm 46.35$ & $0.000^{\star *}$ & $0.000^{* *}$ & $0.000^{* *}$ \\
\hline
\end{tabular}

P1-Control versus Preoperative; P2-Control versus Postoperative; P3-Preoperative versus postoperative *indicate significant change at P0.05); **indicate significant change at $p<0.01 ;{ }^{* *}$ indicate significant change at $p<0.01$. NS means non-significant $(p>0.05)$.

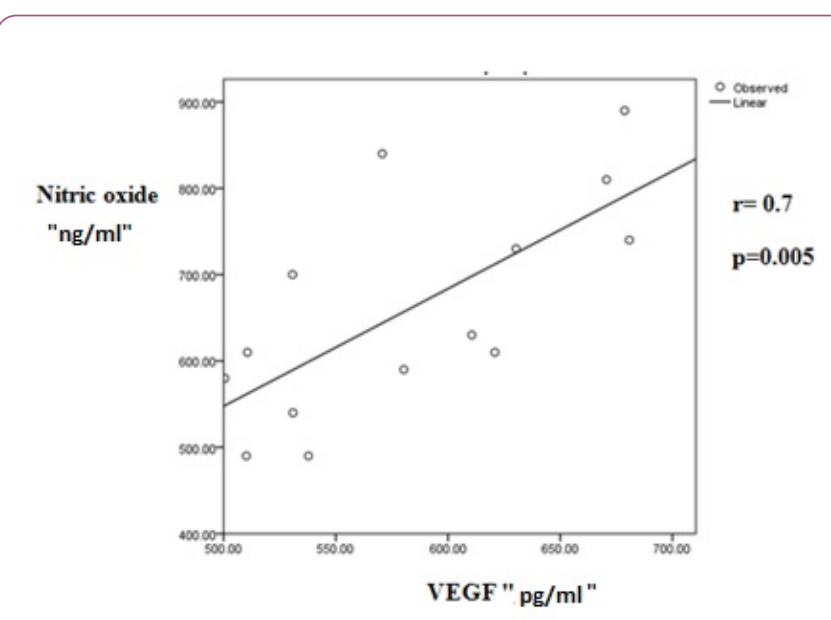

Figure 1 Positive correlation between the plasma levels of nitric oxide and VEGF.

\section{Discussion}

VEGF production is increased in cases of hypoxia by myocytes, smooth muscle cells and endothelial cells [24]. The findings of our study revealed significant higher plasma VEGF preoperatively when compared with the control group, $(p<0.01)$. In agreement with this finding Chung et al. [23], who reported significant elevations in the plasma levels of VEGF among the patients with chronic myocardial infarction "MI" (defined as $\mathrm{Ml}>3$ months previously) when compared with both the control group and patients with acute myocardial infarction suggesting that the activation of angiogenesis in patients with chronic ischemic heart disease may be a delayed response.

To the best of our knowledge, very few studies could be traced in literature regarding the effect of CABG on the plasma levels of VEGF late postoperatively, Although, many studies have been done to evaluate the effect of CABG on the plasma levels of VEGF intraoperatively and early post-CABG within hours, such as, a study done by Burton et al. [25], to test the ability of ischemia, occurring during CABG, to induce VEGF production, found 24 and $48 \mathrm{~h}$ post-CABG, plasma levels of VEGF were significantly elevated by approximately 17.5 and 48.5 fold respectively concluding that the myocardial ischemia secondary to be a potent stimulator of VEGF production and another study done by Denizot et al. [26] to determine whether plasma angiogenic growth factor levels are altered during and after cardiac surgery and reported that plasma VEGF values increased significantly at the 6th and 24th postCABG hours. The correlations of plasma levels of VEGF in patients with coronary artery disease and especially after revascularization intervention are largely unknown [27]. The present study showed significant decrease in the plasma levels of VEGF when repeated measurement three month after the CABG $(p<0.01)$, that could be explained by the decreased requirement of the cardiac muscle for angiogenesis following the CABG because of its revascularization effect which decreases the cardiac ischemia. Our findings were in disagreement with Ramos et al. [27] who found significant lower plasma levels of VEGF among coronary artery disease patients versus the control group and concluded that plasma VEGF levels in those patients progressively increase after revascularization by percutaneous coronary intervention $(\mathrm{PCl})$ during one-year reaching the levels observed in controls.

The VEGF-mediated angiogenesis requires NO production from activated eNOS. Activation of eNOS by VEGF involves several pathways including Akt/ protein kinase B "PKB", $\mathrm{Ca}^{2+}$ / calmodulin, and protein kinase $C$. The NO mediated VEGF production can be controlled by hypoxia inducible factor 1"HIF-1" and hemeoxygenase 1 "HO-1" activity, and the VEGFmediated NO production by eNOS can be also changed by HIF-1 and HO-1 activity, depending upon the amount of produced NO [28]. The results of the present study showed significant higher plasma NO preoperatively when compared with the control group, $(p<0.01)$, with significant decrease in its plasma levels when repeatedly measured three month after the CABG $(p<0.01)$. Also, there was positive correlation between the plasma levels of VEGF and NO ( $r=0.07, p=0.005)$, confirming the VEGF-induced NO production. In agreement 
with this finding, a study done by Papapetropoulos et al. [29], who reported that NO facilitates the VEGF-induced proliferation in human ECs. NO has been also proposed to be a prime mediator of delayed ischemic preconditioning [30].

In particular, bFGF is strongly angiogenic and may act in synergism with VEGF [31]. It mediates VEGF synthesis in the endothelial cells of forming capillaries through autocrine mechanism participating to angiogenesis [32]. The findings of the present study revealed significant higher plasma bFGF preoperatively when compared with the control group $(p<$ 0.01). In agreement with this finding a study done by Padua et al. [33] who found that bFGF increases the cardiac resistance to, and improves the functional recovery from ischemic heart injury. Also, a study done by Yanagisawa-Miwa and coworkers [34], have shown that intracoronary injection of bFGF one week after infarction in a canine experimental infarct model, stimulated angiogenesis, a process proposed to have minimized infarct size and improved cardiac function and that reperfusion after infarction may attenuate the stimulus for neovascularization which explain the significant decreased plasma levels of bFGF serum levels in our study when repeated measurement three month after the CABG $(p<0.01)$. One of the possible mechanisms for bFGF mediated myocardial protection is its ability to stimulate nitric oxide discharge that in turn exhibits a protective effect on the myocardium against ischemia [35].

Copper acts as an obligatory cofactor throughout the angiogenic signaling cascades, to a degree that its deficiency causes neovascularization to decrease. Moreover the progress of various angiogenic pathologies (e.g. diabetes, cardiac hypertrophy and ischemia) can be followed by measuring serum copper levels, which are being increasingly investigated as a useful prognostic marker [36]. In the present study there were significant higher plasma levels of copper preoperatively when compared with the control group $(p<0.01)$, with significant decreased plasma levels when repeated measurement three month after the CABG $(p<0.01)$. It has been demonstrated that activation of HIF-1 $\alpha$ transcription activity requires copper, copper depletion results in inactivation of HIF-1 $\alpha$ and in addition, suppression of VEGF production [37].

\section{Conclusion}

The present study demonstrates that VEGF, bFGF, copper and NO levels as angiogenic biomarkers assume an imperative part in the formation of coronary collateral circulation via angiogenesis in patients with chronic ischemic heart disease and the decrease in their plasma levels post-CABG demonstrate the best possible coronary reperfusion impact of CABG with abatement the requirement for coronary collaterals formation. This indicates the possible benefits of using such markers as an adjuvant therapy in patients with chronic ischemic heart disease in whom the CABG is contraindicated. Also, the present study revealed a positive correlation between the plasma levels of VEGF and NO, confirming the VEGF-induced NO production.

\section{Funding}

This research received no specific grant from any funding agency in the public, commercial, or not-for-profit sectors.

\section{Ethical approval}

The Research Committee at Faculty of Medicine, Assiut University approved this study (IRB00008718 20/01/016).

\section{Acknowledgments}

We would like to acknowledge the team work of the Metabolic and Genetic Disorders Unit, faculty of medicine, Assiut University, where the laboratory work of this study has been done.

\section{References}

1. Diodato M, Chedrawy EG (2014) Coronary Artery Bypass Graft Surgery: The Past, Present, and Future of Myocardial Revascularisation. Surgery Research and Practice 1-6.

2. Fukuda $S$, Yoshii $S$, Kaga $S$, Matsumoto $M$, Kugiyama $K$, et al. (2004) Angiogenic strategy for human ischemic heart disease: Brief overview. Molecular and Cellular Biochemistry 264: 143-149.

3. Fujita M (1995) Salutary effects of myocardial ischemia in coronary artery disease. Int J Cardiol 52: 197-202.

4. Schaper W, Ito WD (1996) Molecular mechanisms of coronary collateral vessel growth. Cric Res 79: 911-919.

5. Jacobs J (2007) Combating cardiovascular disease with angiogenic therapy. Drug Discovery Today 12: 1040-1045.

6. Pellet-Many C, Frankel P, Jia H, Zachary I (2008) Neuropilins: structure, function and role in disease. Biochem J 411: 211-226.

7. Senger DR, Galli SJ, Dvorak AM, Perruzzi CA, Harvey VS (1983) Tumor cells secrete a vascular permeability factor that promotes accumulation of ascites fluid. Science 219: 983-985.

8. Ferrara N, Henzel WJ (1989) Pituitary follicular cells secrete a novel heparin-binding growth factor specific for vascular endothelial cells. Biochem. Biophys. Res. Commun 161 : 851-858.

9. Benjamin LE, Golijanin D, Itin A, Pode D, Keshet E (1999) Selective ablation of immature blood vessels in established human tumors follows vascular endothelial growth factor withdrawal. J Clin Invest 103 : 159-165.

10. Gerber HP, McMurtrey A, Kowalski J, Yan M, Keyt BA, et al. (1998) Vascular endothelial growth factor regulates endothelial cell survival through the phosphatidylinositol 3'-kinase/Akt signal transduction pathway. Requirement for Flk-1/KDR activation. J Biol Chem 273: 30336-30343.

11. Nagy JA, Benjamin L, Zeng H, Dvorak AM, Dvorak HF (2008) Vascular permeability, vascular hyperpermeability and angiogenesis. Angiogenesis 11: 109-119.

12. Yu J, de Muinck ED, Zhuang Z, Drinane M, Kauser K, et al. (2005) Endothelial nitric oxide synthase is critical for ischemic remodeling, mural cell recruitment, and blood flow reserve. Proc Natl Acad Sci USA 102: 999-1004. 
13. Folkman J (1995) Tumorangiogenesis. In: Mendelsohn J, Howley P, Israel M, Liotta M (Eds). The Molecular Basis of Cancer. Philadelphia: W.B. Saunders 206-232.

14. Lowndes SA, Harris AL (2005) The Role of Copper in Tumour Angiogenesis. J Mammary Gland Biol Neoplasia 10: 299-310.

15. McAuslan BR, Reilly W (1980) Endothelial cell phagokinesis in response to specific metal ions. Exp Cell Res 130: 147-157.

16. Hu GF (1998) Copper stimulates proliferation of human endothelial cells under culture. J Cell Biochem 69: 326-335.

17. Gruetter CA, Barry BK, McNamara DB, Gruetter DY, Kadowitz PJ, et al. (1979) Relaxation of bovine coronary artery and activation of coronary arterial guanylate cyclase by nitric oxide nitroprusside and a carcinogenic nitrosoamine. J Cyclic Nucleotide Res 5 : 211-224.

18. Furchgott RF, Zawadzki JV (1980) The obligatory role of endothelial cells in the relaxation of arterial smooth muscle by acetylcholine. Nature 288: 373-376.

19. Ignarro LJ (1996) Physiology and pathophysiology of nitric oxide. Kidney Int Suppl 55: 2-5.

20. Mayer B (1994) Nitric oxide / cyclic GMP-mediated signal transduction. Ann N Y Acad Sci 733: 357-364.

21. Knowles RG, Moncada S (1994) Nitric oxide synthases in mammals. Biochem J 298: 249-458.

22. Jelkmann $W$ (2001) Pitfalls in the measurement of circulating vascular endothelial growth factor. Clin Chem 47: 617-623.

23. Chung NA, Belgore CL, Blann AD, Lip GY (2002) Angiogenesis in myocardial infarction; An acute or chronic process? Eur Heart J 23: 1604-1608.

24. Levy AP, Levy NS, Wegner S, Goldberg MA (1995) Transcriptional regulation of the rat vascular endothelial growth factor gene by hypoxia. J Biol Chem 270: 1333-1340.

25. Burton PB, Owen VJ, Hafizi S, Barton PJ, Carr-White G, et al. (2000) Vascular endothelial growth factor release following coronary artery bypass surgery: extracorporeal circulation versus'beating heart'surgery. Eur Heart J 21: 1708-1713.

26. Denizot Y, Guglielmi L, Cornu E, Nathan N (2003) Alterations in plasma angiogenic growth factor concentrations after coronary artery bypass graft surgery: relationships with post-operative complications. Cytokine 24: 7-12.

27. Ramos C, Napoleão P, Selas M, Freixo C, Crespo A, et al. (2014) Prognostic Value of VEGF in Patients Submitted to Percutaneous Coronary Intervention. Disease Markers 1-7.

28. Kimura H, Esumi H (2003) Reciprocal regulation between nitric oxide and vascular endothelial growth factor in angiogenesis. Acta Biochimica Polonica 50: 49-59.

29. Papapetropoulos A, García-Cardeña G, Madri J, Sessa W (1997) Nitric oxide production contributes to the angiogenic properties of vascular endothelial growth factor in human endothelial cells. J Clin Invest 100: 3131-3139.

30. Carden DL, Granger ND (2000) Pathophysiology of ischaemiareperfusion injury. J Pathol 190: 255-266.

31. Losordo DW, Dimmeler S (2004) Therapeutic Angiogenesis and Vasculogenesis for Ischemic Disease Part I: Angiogenic Cytokines. Circulation 109: 2487-2491.

32. Seghezzi G, Patel S, Ren CJ (1998) Fibroblast growth factor-2 (FGF-2) induces vascular endothelial growth factor (VEGF) expression in the endothelial cells of forming capillaries: an autocrine mechanism contributing to angiogenesis. J Cell Biol 141: 1659-1673.

33. Padua R, Sethi R, Dhalla NS, Kardami E (1995) Basic fibroblast growth factor is cardioprotective in ischemia-reperfusion injury. Mol Cell Biochem 143: 129-135.

34. Yanagisawa-Miwa A, Uchida $Y$, Nakamur $F$ (1992) Salvage of infarcted myocardium by angiogenic action of basic fibroblast growth factor. Science 257: 1401-1403.

35. Cuevas P, Carceller F, Martinez-Coso V (1999) Cardioprotection from ischemia by fibroblast growth factor: role of inducible nitric oxide synthase. Eur J Med Res 4: 517-524.

36. Urso E, Maffia M (2015) Behind the Link between Copper and Angiogenesis: Established Mechanisms and an Overview on the Role of Vascular Copper Transport Systems. J Vasc Res 52:172-196.

37. Feng W, Ye F, Xue W, Zhou Z, Kang YJ (2009) Copper regulation of hypoxia-inducible factor-1 activity. Mol Pharmacol 75: 174-182. 